\title{
Qualité germinative des semences de pois. II. - Suivi conductimétrique des graines au cours de leur développement ; incidence du relargage des nutriments sur la croissance des plantules
}

Zohreh RACHIDIAN \& Yvon LE DEUNFF

INA Paris - Grignon, Laboratoire de Physiologie végétale, 16, rue Claude-Bernard, F 75231 Paris Cedex 05

RÉSUMÉ

\begin{abstract}
Au cours du développement des semences de pois, Pisum sativum var. "Finale ", la conductivité de leur lessivat aqueux est suivie, après un trempage de $24 \mathrm{~h}$ à $20^{\circ} \mathrm{C}$, à l'aide d'un milliampèremètre de type ASA610. L'évolution de la conductivité est fortement liée aux électrolytes qui parviennent aux semences immatures. Le relargage de ces éléments, soit dans le milieu d'imbibition des graines mises à germer à leur teneur en eau initiale, soit dans le substrat utilisé pour la croissance des plantules, peut, au moins en partie, expliquer l'évolution de la capacité germinative des semences prélevées plus ou moins hydratées ainsi que la croissance retardée des plantules. La déshydratation des semences immatures, réalisée à température modérée, permet une bonne germination ainsi que l'élimination du mode de croissance retardée. Nous montrons ainsi que les semences immatures gardent une mémoire importante des événements subis sur la plante-mère et qu'un séchage modéré élimine la plupart des freins mis en place au niveau germination et au niveau croissance. Si le battage des gousses très hydratées était possible, on pourrait cueillir les semences plus tôt et les sécher sans trop altérer leur qualité.
\end{abstract}

Mots clés additionnels : Germination, plantules anormales, séchage, Pisum sativum $L$. and effect of nutrient leakage on seedling growth.

Seeds of Pisum sativum cv. Finale at different stages of development were soaked in water for $24 \mathrm{~h}$ at $20{ }^{\circ} \mathrm{C}$ and electrolyte leakage under these conditions was measured with an ASA 610 milliammeter. The amount of leakage was strongly connected with the electrolytes reaching the immature seeds. Loss of these electrolytes into the seed imbitition medium or seedling culture medium partly explained the poor germination characteristics and delayed seedling growth of immature seeds. Drying immature seeds at moderate temperature improved their germination capacity and seedling growth. Accordingly, immature seeds seemed to be much affected by their previous history on the mother-plant. Drying them eliminated most of these acquired blocks to germination and seedling growth. If it were possible to thresh pods when the seeds are still hydrated, the seeds could then be dried without loss of quality and with a corresponding gain in time.

Additional key words : Germination, abnormal seedlings, drying, Pisum sativum $L$.

\section{INTRODUCTION}

Nous avons montré précédemment (RACHIDIAN \& LE DEUNFF, 1986) les rapports étroits existant entre la gousse et ses graines au cours de leur développement. La période de sénescence de la gousse a pu être décomposée en 2 étapes très nettement délimitées dans le temps. Les difficultés de germination des semences récoltées immatures peuvent s'expliquer, soit par une trop forte concentration en acide abscissique (ABA) dans la graine, soit par l'existence de composé(s) vola- til(s) pouvant inhiber certaines réactions hydrolytiques. Nous proposons ici une $3^{\mathrm{e}}$ interprétation qui tient compte de la perte d'éléments nutritifs par les semences immatures quand elles sont placées sur un milieu d'imbibition. L'origine de ces nutriments est à rechercher dans les événements mis en évidence lors du remplissage des graines au cours de leur développement. Ainsi, sont connus le déchargement de la sève (à partir du phloème présent dans le tégument séminal) dans l'apoplasme du parenchyme aérifère chez le soja (THORNE, 1981) ainsi que le déversement de cette 
sève dans l'espace libre entre tégument et embryon : ceci est décrit chez la fève (WOLSWINKEL \& AMMERLAAN, 1983) et le soja (THORNE \& RAINBIRD, 1983). On peut penser que des phénomènes analogues existent chez le pois. Les métabolites ainsi déversés sucres et acides aminés entre autres - et les ions minéraux sont repris au niveau des cellules externes des cotylédons à l'aide de transporteurs spécifiques si leur concentration est faible ou par un système diffusif si leur concentration est élevée ; ainsi en est-il chez le soja (THORNE, 1982; BENNETT \& SPANSWICK, 1983).

La plupart de ces nutriments transportés par la sève sont des électrolytes et une technique simple, telle la conductimétrie, doit permettre de connaître leur quantité globale à tout moment du développement des semences (BEDFORD \& MATTHEWS, 1976). Il est permis de penser que ces électrolytes et les composés neutres, nutriments essentiels de la graine, s'ils sont perdus par un lessivage trop important et s'ils ne sont pas réabsorbés par la semence, vont faire défaut pour assurer une bonne germination des graines ainsi qu'une croissance correcte des plantules s'il y a germination. Nous présentons ici les résultats relatifs à l'évolution de la conductivité des lessivats des graines plus ou moins hydratées de pois protéagineux. Ces données sont mises en relation avec la germination et la croissance des plantules normales. Nous montrons enfin que la qualité des semences est fortement conditionnée par la teneur en eau initiale avant la germination et que le séchage tend à améliorer cette qualité.

\section{MATÉRIEL ET MÉTHODES}

Le matériel végétal est celui déjà décrit (RACHIDIAN \& LE DeUNFF, 1986) mais, ici, seules les graines récoltées en 1984 sont utilisées. La germination est traduite en capacité germinative à $20^{\circ} \mathrm{C}$, à la lumière $(8 \mathrm{~h} / 16 \mathrm{~h})$ et en faculté germinative : celle-ci correspond à la valeur de la capacité germinative diminuée du pourcentage de plantules anormales. Les plantules anormales sont décrites dans le Manuel de l'ISTA (1979).

\section{A. Lessivage des électrolytes et leur mesure}

Immédiatement après l'extraction manuelle des graines de leur gousse, on procède à la mesure de la conductivité des lessivats aqueux de 400 individus, à l'aide d'un milliampèremètre de type ASA 610 (STEERE et al., 1981). Les résultats obtenus sont ainsi traduits en terme de courant et non pas en Siemens comme le permet un conductimètre classique.

L'appareil est constitué d'une sonde de mesure comportant 100 paires d'électrodes, chaque paire servant à mesurer la conductivité du milieu aqueux entourant une seule graine, après des temps variés de trempage. Un plateau en plastique comportant 100 alvéoles de même volume est plongé dans de l'eau déminéralisée ; on égalise les niveaux d'eau à l'aide d'une plaque ultra plane. Les graines sont disposées dans le plateau à raison d'une par alvéole. Le trempage des graines dure $24 \mathrm{~h}$ à la température de $20^{\circ} \mathrm{C}$.
$\mathrm{Au}$ terme de cette période les électrodes sont plongées dans les alvéoles correspondantes sans ôter les graines. La lecture de la conductivité est réalisée électroniquement selon un programme de balayage de la première à la dernière alvéole : les résultats, obtenus en quelques secondes, sont transcrits automatiquement sur une feuille de papier dans l'ordre de lecture. Chaque graine mise à tremper est ainsi individualisée par un chiffre représentant la quantité d'électrolytes relâchée en $24 \mathrm{~h}$ dans de l'eau déminéralisée.

Les résultats présentés ici sont obtenus à l'aide de 4 plateaux de 100 graines chacun. La mise en trempage des graines est légèrement décalée entre chaque plateau de manière à réaliser les mesures après un temps réel de $24 \mathrm{~h}$. Les valeurs moyennes de conductivité en $\mu \mathrm{A}$ sont rapportées soit à une graine, soit à $1 \mathrm{~g}$ de matière sèche initiale (passage des graines à $130{ }^{\circ} \mathrm{C}$ durant $24 \mathrm{~h}$ ).

\section{B. Séchage ventilé et faculté germinative}

Des graines prélevées dans leur gousse, à 4 moments de leur développement $-108,115,119$ et $128^{\mathrm{e}} \mathrm{j}$ après semis - sont placées dans des étuves ventilées à 15 , 20 et $35^{\circ} \mathrm{C}$ jusqu'à l'obtention d'une teneur en eau de 14 p. 100 . Elles sont ensuite mises à germer pendant $12 \mathrm{j}$ dans les conditions décrites précédemment (RACHIDIAN \& LE DEUNFF, 1986). Au $12^{\mathrm{e}} \mathrm{j}$, on effectue le dénombrement des graines mortes ou non germées, des plantules anormales et des plantules normales.

\section{Croissance des plantules}

A l'issue de $12 \mathrm{j}$ de croissance, seules les plantules normales sont disséquées en 3 parties : les cotylédons, l'épicotyle et la racine; les 2 dernières sont passées à l'étuve à $130{ }^{\circ} \mathrm{C}$ durant $24 \mathrm{~h}$, puis pesées. Les résultats présentés correspondent à la matière sèche moyenne de l'épicotyle et de la racine, la somme des 2 valeurs correspondant à la plantule entière sans les cotylédons.

Deux expériences ont été réalisées : l'une avec des graines immatures à forte teneur en eau, mises immédiatement à germer, l'autre avec des graines immatures s'étant déshydratées à la température du laboratoire. Le rapport de la matière sèche des plantules (racine et épicotyle sans les cotylédons) et de celle des graines au moment du semis permet de comparer l'efficacité de croissance des plantules en fonction de la taille et de l'état de développement des graines sur la plante-mère.

\section{RÉSULTATS}

\section{A. Evolution de la conductivité des lessivats de graines}

La conductivité par graine (fig. 1) augmente parallèlement à l'augmentation de matière fraîche des graines (cf. fig. 1B, RACHIDIAN \& LE DEUNFF, 1986). On observe un optimum au $119^{\mathrm{e}} \mathrm{j}$ après semis, puis une baisse très rapide, la conductivité étant de $25 \mu \mathrm{A}$ quand la teneur en eau de la graine est de 40 p. 100. 


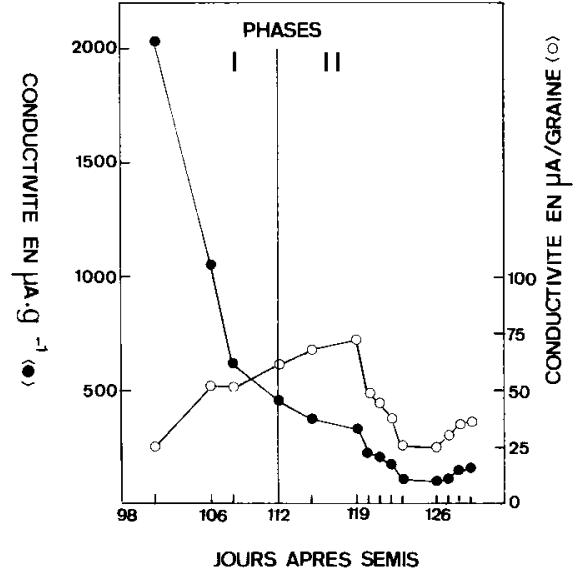

Figure 1

Evolution de la conductivité, à $20^{\circ} \mathrm{C}$, des lessivats de graines de pois protéagineux (Pisum sativum L. var. "Finale ") au cours de leur développement. Les valeurs sont ramenées à une graine $(\bigcirc)$ ou à $1 \mathrm{~g}$ de matière sèche $(\bullet)$.

Conductivity of washings from Pisum sativum seeds, at $20{ }^{\circ} \mathrm{C}$, during development. Values are reported per seed (O) or per $g$ dry weight $(\bullet)$.

Une très légère augmentation est visible lorsque la teneur en eau diminue jusqu'à 19 p. 100 .

Si la conductivité est rapportée à la matière sèche, on note une diminution par étapes qui suit assez étroitement l'évolution de la déshydratation des graines (cf. fig. 1B, RACHIDIAN \& LE DEUNFF, 1986). Plus elles sont initialement hydratées, plus elles relâchent des électrolytes dans un milieu aqueux : l'obtention de la maturité physiologique, au $119^{\text {e }}$ j (57 p. 100 de teneur en eau), se traduit particulièrement au niveau conductivité quel que soit le mode d'expression (fig. 1).

\section{B. Influence du séchage ventilé sur la faculté germi- native}

Le séchage ventilé des graines à 15,20 et $35^{\circ} \mathrm{C}$ permet l'obtention des résultats de germination du tableau 1 .

Les semences très hydratées $(77,20$ p. 100) germent, après séchage, en donnant quelques plantules anormales quelle que soit la température de séchage : à $35^{\circ} \mathrm{C}, 49 \mathrm{p}$. 100 des graines mises à germer meurent. Cette mortalité est encore plus importante quand la teneur en eau est de 62,70 p. 100 . Dès que celle-ci atteint ou est inférieure à 57,35 p. 100 , on observe très peu de plantules anormales et de graines mortes, sauf à la température de séchage de $15^{\circ} \mathrm{C}$. Lorsque les graines sont encore très hydratées, aux alentours de 57 p. 100, un séchage modéré à des températures physiologiques permet une excellente faculté germinative.

\section{Croissance des plantules}

\section{Issues de graines immatures sans séchage}

$\mathrm{Au} 12^{\mathrm{e}} \mathrm{j}$ de croissance, la matière sèche des plantules - sans les cotylédons - est très différente selon le moment du prélèvement des graines mises à germer (fig. 2). L'allure générale de la courbe représentant l'évolution de ces valeurs en fonction de la date de prélèvement est une sigmoïde. La croissance est très faible du 108 au $115^{\mathrm{e}} \mathrm{j}$, période où les graines commencent à acquérir l'aptitude à germer ; elle est ensuite de plus en plus importante jusqu'au $128^{\mathrm{e}} \mathrm{j}$, puis n'évolue plus. La dépression de la germination,

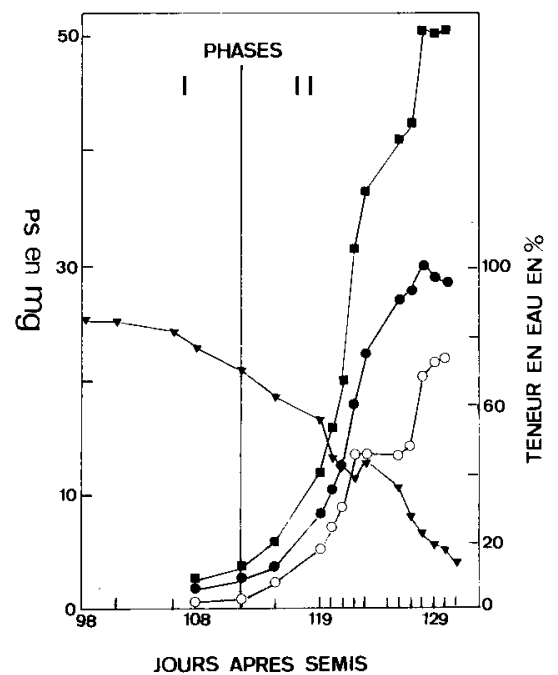

Figure 2

Evolution de la matière sèche des plantules sans cotylédons (ם) et de ses composantes épicotyle $(0)$ et racine $(\bullet)$ de pois protéagineux (Pisum sativum L.) au bout de $12 \mathrm{j}$ de croissance à $20{ }^{\circ} \mathrm{C}$, à la lumière $(8 \mathrm{~h} / 16 \mathrm{~h})$ en fonction des dates de prélèvement des semences sur la plante-mère. On a représenté la déshydratation des graines sur la plante-mère (A).

Dry weight of seedlings without cotyledons (⿴), epicotyl $(\mathrm{O})$ and root $(\bullet)$ in pea (Pisum sativum L.) after twelve days culture at $20{ }^{\circ} \mathrm{C}$, in the light $(8 \mathrm{~h} / 16 \mathrm{~h})$ for various harvest dates throughout development. Seed dehydration on the mother plant is shown as (A).

\section{TABLEAU 1}

Influence de la teneur en eau initiale des graines de pois var. «Finale" sur la germination et l'apparition des plantules anormales obtenues en $10 j$ après séchage ventilé des semences à 15,20 et $35^{\circ} \mathrm{C}$; la teneur en eau est alors de $14 \mathrm{p}$. 100 .

Influence of initial seed moisture content (Pisum sativum var. "Finale") on germination and occurrence of abnormal seedlings obtained in ten days after aerated drying at 15,20 and $35{ }^{\circ} \mathrm{C}$ to a moisture content of $14 \%$.

\begin{tabular}{|c|c|c|c|c|c|c|c|c|c|c|c|c|}
\hline Prélèvement (jours après semis) & \multicolumn{3}{|c|}{108} & \multicolumn{3}{|c|}{115} & \multicolumn{3}{|c|}{119} & \multicolumn{3}{|c|}{128} \\
\hline Teneur en eau en $\%$ & \multicolumn{3}{|c|}{77,20} & \multicolumn{3}{|c|}{62,70} & \multicolumn{3}{|c|}{57,35} & \multicolumn{3}{|c|}{22,70} \\
\hline Température de séchage en ${ }^{\circ} \mathrm{C}$ & 15 & 20 & 35 & 15 & 20 & 35 & 15 & 20 & 35 & 15 & 20 & 35 \\
\hline Capacité germinative & 97,5 & 95 & 51 & 98,5 & 97,5 & 11 & 97,5 & 100 & 99,5 & 100 & 99 & 98 \\
\hline Plantules anormales en $\%$ & 4,5 & 3,5 & 4 & 4,5 & 7 & 3,5 & 5 & 0,5 & 0 & 1,5 & 1,5 & 1,5 \\
\hline Graines mortes en $\sigma_{0}$ & 2,5 & 5 & 49 & 1,5 & 2,5 & 89 & 2,5 & 0 & 0,5 & 0 & 1 & 2 \\
\hline Faculté germinative & 93 & 91,5 & 47 & 94 & 90,5 & 7,5 & 92,5 & 99,5 & 99,5 & $\cdot 98,5$ & 97,5 & 96,5 \\
\hline
\end{tabular}


montrée précédemment (RACHIDIAN \& LE DEUNFF, 1986), ne se retrouve pas au niveau de la croissance pondérale des plantules issues des graines qui ont surmonté l'inhibition à germer. Par contre, des freins à la croissance existent puisque celle-ci est très retardée, le mode " normal " n'étant atteint qu'au $128^{\mathrm{e}} \mathrm{j}$, bien que l'ensemble des graines mises à germer dès le $121^{\mathrm{e}} \mathrm{j}$ donne naissance à des plantules.

Cette croissance retardée des plantules normales est mesurable aussi bien chez la racine que chez l'épicotyle (fig. 2). A partir du $115^{\mathrm{e}} \mathrm{j}$, les racines et les épicotyles croissent d'autant plus vite que la date de prélèvement est plus tardive. L'arrêt de croissance observé chez les épicotyles du 122 au $127^{\mathrm{e}} \mathrm{j}$ après le semis est sans doute lié aux fluctuations climatiques en cette période.

La croissance retardée, affectant la racine et l'épicotyle, suggère que la vitesse d'hydrolyse des réserves cotylédonaires est variable selon l'état initial de développement des graines sur la plante-mère. Afin d'éliminer l'influence du facteur taille des graines - donc poids de matière sèche - sur la production de matière sèche des plantules, au $12^{\mathrm{e}} \mathrm{j}$ de croissance, on utilise le rapport matière sèche plantules/matière sèche initiale des graines au semis : ce rapport reflète la migration des hydrolysats chez des jeunes plantes de même âge. Il est très faible (tabl. 2) au $115^{\mathrm{e}} \mathrm{j}$, puis il augmente progressivement jusqu'au prélèvement du $130^{\mathrm{e}} \mathrm{j}$. Lorsque la capacité germinative atteint 100 p. 100, au $121^{\mathrm{e}} \mathrm{j}$, ce rapport continue à croître, suggérant que si les freins à la germination sont éliminés il existe encore des freins à la croissance des plantules (tabl. 2).

\section{Issues de graines immatures déshydratées au laboratoire}

Lorsque les graines prélevées immatures sont laissées dans les conditions ambiantes du laboratoire,

\section{TABLEAU 2}

Evolution de la matière sèche des graines de pois protéagineux var. "Finale " au cours du développement, en 1984, et des plantules issues de leur germination après $12 j$ de croissance dà $20{ }^{\circ} \mathrm{C}$, à la lumière $(8 \mathrm{~h} / 16 \mathrm{~h})$. Le nombre entre parenthèses correspond à la capacité germinative des semences. Le témoin est relatif à des semences récoltées en 1983, l'expérience ayant lieu 6 mois après la récolte.

Change in seed dry weight through development, in 1984, and in seedling dry matter after 12 days of culture at $20{ }^{\circ} \mathrm{C}$, in the light $(8 \mathrm{~h} / 16 \mathrm{~h})$. Numbers between brackets are germination capacities. The control corresponds to seeds harvested in 1983, experimentation being realized six months after harvest.

\begin{tabular}{ccccc}
\hline \hline $\begin{array}{c}\text { Moment du } \\
\text { prélèvement } \\
\text { en j } \\
\text { après le semis }\end{array}$ & $\begin{array}{c}\text { Teneur } \\
\text { en eau } \\
\text { en } \%\end{array}$ & $\begin{array}{c}\text { Matière sèche } \\
\text { en mg }\end{array}$ & $\begin{array}{c}\text { MS plantule/ } \\
\text { MS graine } \\
\text { en \% }\end{array}$ \\
\cline { 3 - 4 } 115 & $63,0(52)$ & 182 & 6 & 3,2 \\
119 & $56,9(44)$ & 218 & 12 & 5,5 \\
120 & $45,7(33,5)$ & 222 & 16 & 7,2 \\
121 & $43,6(100)$ & 220 & 20 & 9,1 \\
122 & 39,0 & 220 & 32 & 14,5 \\
123 & $44,5(100)$ & 230 & 36,5 & 15,9 \\
126 & $35,7(98,5)$ & 240 & 41 & 17,1 \\
127 & $28,2(100)$ & 250 & 42,5 & 17 \\
130 & $23,0(100)$ & 250 & 51 & 20,4 \\
Témoin & $11,5(100)$ & 281 & 53 & 18,9 \\
\hline
\end{tabular}

elles se déshydratent en équilibrant leur teneur en eau avec l'humidité de l'air. La capacité germinative des graines prélevées précocement est alors fortement améliorée (tabl. 2 et 3). La croissance pondérale des plantules est elle aussi fortement augmentée par rapport à celle des plantules issues des graines immatures hydratées, de même âge : les rapports de matière sèche plantule/graine sont aussi plus importants (tabl. 3). L'hydrolyse des réserves cotylédonaires et la migration des hydrolysats dans la plantule sont fortement améliorées par une déshydratation lente et préalable des graines immatures.

\section{TABLEAU 3}

Influence de la teneur en eau initiale des semences de pois (Pisum sativum, var. "Finale ») sur la croissance pondérale au $12^{e} j$ des plantules (matière sèche) obtenues après que les semences immatures aient équilibré leur teneur en eau avec l'atmosphère ambiante du laboratoire à $20{ }^{\circ} \mathrm{C}$. Les nombres entre parenthèses sont les valeurs des capacités germinatives.

Influence of initial seed moisture content on seedling growth (in dry matter) after twelve days culture, when the immature seeds have equilibrated their moisture content with the ambient laboratory air at $20^{\circ} \mathrm{C}$. Numbers between brackets are germination capacities.

\begin{tabular}{|c|c|c|c|c|}
\hline \multicolumn{2}{|c|}{ Teneur en eau $(\%)$} & \multicolumn{2}{|c|}{ Matière sèche en mg } & \multirow{2}{*}{$\begin{array}{c}\text { MS plantule } \\
\text { MS graine } \\
\text { en } \%\end{array}$} \\
\hline Récolte & Germination & Graine & Plantule & \\
\hline 77,7 & $12,8(99)$ & 110,3 & 42,2 & 38,25 \\
\hline 62,7 & 13,4 & 191,5 & 56,6 & 29,55 \\
\hline 56,8 & $13,5(96)$ & 197,3 & 62,8 & 31,82 \\
\hline 43,6 & 13,6 & 213,2 & 58,9 & 27,62 \\
\hline 28,2 & 13,3 & 240,2 & 63,3 & 26,35 \\
\hline 23 & $13,4(97)$ & 262,4 & 71,8 & 27,36 \\
\hline
\end{tabular}

\section{DISCUSSION}

\section{A. Conductivité des lessivats}

La conductivité des lessivats de graines plus ou moins hydratées décroît en suivant l'évolution de la déshydratation des semences décrites précédemment (RACHIDIAN \& LE DEUnFF, 1986). Sur une autre variété de pois, en utilisant un conductimètre, BEDFORD \& MATTHEWS, en 1976, n'ont pas trouvé les phases que nous décrivons, mais ils ont constaté une baisse régulière de la conductivité, celle-ci atteignant un niveau constant lorsque le grain atteint son optimum de matière fraîche.

Le tégument séminal, le cotylédon et l'axe de graines immatures de pois relâchent dans un milieu aqueux de nombreux nutriments dont des acides aminés : leur relargage est d'autant plus abondant que les graines sont plus immatures (DE RUITER et al., 1984), la composition qualitative du lessivat étant alors voisine de celle de la sève (MurRay \& KenNEDY, 1980). Les autres solutés lessivés sont des sucres, des phénols et des ions minéraux, ces derniers étant seuls capables de rendre compte de la forte conductivité observée.

Les résultats relatifs au lessivage des ions minéraux par les semences immatures sont rares : chez le haricot, $\mathrm{K}^{+}$représente 35 p. 100 de l'osmolalité totale du tégument séminal, soit $180 \mathrm{mM}$ (PATRICK, 1984). 
Chez les graines mûres, à la récolte, on connaît mieux les ions relâchés : ce sont essentiellement $\mathrm{Ca}^{++}$, $\mathrm{Mg}^{++}, \mathrm{Na}^{+}$(GIVELBERG et al., 1984) et surtout $\mathrm{K}^{+}$ (SAMAD \& PEARCE, 1978 ; LOOMIS \& SMITH, 1980 ; Mc KERSIE \& STINSON, 1980) : leur abondance dans le milieu de trempage est corrélée positivement à l'évolution de l'hydratation des graines. Chez le lupin, une forte corrélation existe ainsi entre la conductivité mesurée et la quantité de $\mathrm{K}^{+}$relarguée $(\mathrm{r}=0,97$; TOUBOUL, 1984).

La fuite importante de solutés et d'ions minéraux essentiels peut gêner la germination, donc la croissance des plantules, dans la mesure où des transporteurs actifs d'absorption ne sont pas opérants après le lessivage. Ainsi le potassium, dont on connaît le rôle essentiel pour la germination et la croissance (CHAUSSAT, 1968 ; LE DEUNFF, 1971 ; LEIGH \& WYNJONES, 1984), est relargué très rapidement - 10 premières heures - par des graines de radis (COCUCCI \& CoCuCCI, 1977 ; Petruzelli et al., 1982) puis réabsorbé à l'aide d'une ATPase activée par $\mathrm{K}^{+}$. Il en est de même chez Phacelia tanacetifolia (PARDI et al., 1980).

A la lumière de nos résultats préliminaires, il apparaît nécessaire d'approfondir la composition en ions minéraux des lessivats des graines immatures, puis d'aborder l'étude des transporteurs actifs de reprise de ces ions et de leur activité au cours du développement, cette étude devant être réalisée in situ et in vitro. Dans le second cas, un grand intérêt sera porté à la pression osmotique du milieu qui est un facteur limitant de l'absorption des solutés par les cotylédons lorsqu'elle est faible (WOLSWINKEL \& AMMERLAAN, 1985) et qui empêche la germination précoce lorsqu'elle est élevée (OBENDORF \& WETTLAUFER, 1984).

\section{B. Séchage ventilé et faculté germinative}

Un séchage ventilé à des températures physiologiques entraîne une capacité germinative élevée même lorsque les graines sont très immatures. Des résultats voisins ont été obtenus chez les céréales (EvANS et al., 1975) et les axes de haricot (LONG et al., 1981 ; DAS GUPTA \& BEWLEY, 1982) chez lesquels la dessication entraîne le passage irréversible d'un programme développement à un programme germination. Lorsque les graines de pois ont une teneur en eau proche de celle correspondant à leur maturité physiologique (57 p. 100) un séchage ventilé, quelle que soit la température, au moins jusqu'à $35^{\circ} \mathrm{C}$, permet l'obtention d'une très forte capacité germinative, d'un minimum de plantules anormales donc d'une très forte faculté germinative.

\section{Croissance des plantules}

Les plantules issues de graines immatures non séchées (fig. 2 et tabl. 2) ont une croissance retardée affectant racine et épicotyle. Elle est surtout observable quand les graines mises à germer relarguent encore des électrolytes en grande quantité (fig. 1), du 108 au $119^{\mathrm{e}} \mathrm{j}$. Les 2 interprétations données précédemment pour expliquer la dépression de la capacité germinative (RACHIDIAN \& LE DEUNFF, 1986), accumulation d'ABA et intervention de composés volatils, restent valables pour expliquer les résultats obtenus pour la croissance des plantules ; on peut y ajouter une composante trophique liée au lessivage. Lorsque les freins à la germination sont élevés (121 $\mathrm{e}$ j après le semis), des freins à la croissance persistent encore quelques jours : leur origine peut être essentiellement trophique.

Les plantules issues de graines immatures déshydratées au laboratoire montrent une croissance très nettement améliorée par rapport à celles issues de graines immatures hydratées. La déshydratation des graines immatures permet de surmonter les freins à la germination (tabl. 1) et les freins à la croissance (tabl. 3). La déshydratation, réalisée avec ou sans ventilation, permet le passage d'un programme développement des graines - sans doute purement anabolique - à un programme germination puis croissance hétérotrophe, les 2 nécessitant un intense catabolisme préparatoire (ADAMS \& RINNE, 1980 ; MISRA \& BEWLEY, 1985).

\section{Qualité des semences de pois protéagineux var. «Finale »}

Ces résultats mettent en évidence que la qualité des semences et la croissance des plantules qui en sont issues sont liées étroitement au développement des graines sur la plante-mère ; elles conservent une « mémoire » très forte des influences reçues, l'ABA, les composés volatils et les nutriments entrant dans cette catégorie. Un séchage modéré permet d'éliminer les freins - ABA et composés volatils - et induit sans doute le fonctionnement de transporteurs actifs des solutés essentiels lessivés au tout début de l'imbibition. Si le battage des gousses très hydratées était possible sans abîmer les semences, on pourrait préconiser une récolte beaucoup plus précoce sans risques d'obtention d'anormaux et de plantules chétives (tabl. 3). Il reste beaucoup à faire pour mieux comprendre les liens existant entre le développement des semences et leur qualité. Sur le plan des mécanismes moléculaires, ce travail est en cours de réalisation.

Reçu le 15 octobre 1985. Accepté le 13 mars 1986.
Adams C. A., Rinne R. W., 1980. Moisture content as a controlling factor in seed development and germination. Int. Rev. Cytol., 68, $1-8$.

Bedford L. V., Matthews S., 1976. The effect of seed age at harvest on the germinability and quality of heat-dried seed peas. Seed Sci. and Technol., 4, 275-286.
Bennett A. B., Spanswick R. M., 1983. Derepression of amino-acid $\mathrm{H}^{+}$cotransport in developing soybean embryos. Plant Physiol., 72, $781-786$.

Chaussat R., 1968. Action particulière du potassium sur la croissance et la morphologie des plantules étiolées de blé. Ann. Physiol. vég., 10, 5-16. 
Cocucci S., Cocucci M., 1977. Effect of ABA, GA $\mathrm{And}_{3}$ and on the development of potassium uptake in germinating radish seeds. Plant Sci. Lett., 10, 85-95.

Dasgupta J., Bewley J. D., 1982. Desiccation of axes of Phaseolus vulgaris during development of a switch from a developmental pattern of protein synthesis to a germination pattern. Plant Physiol., 70, 1224-1227.

De Ruiter H., Schuurmans J., Kolloffel C., 1984. Amino-acid leakage from cotyledons of developing and germinating pea seeds. $J$. Plant Physiol., 116, 47-57.

Evans M., Black M., Chapman J., 1975. Induction of hormone sensitivity by dehydration is one positive role for drying in cereal seed. Nature (Lond.), 258, 144-145.

Givelberg A., Horowitz M., Pojlakoff-Mayber A., 1984. Solute leakage from Solanum nigrum seeds exposed to high temperatures during imbibition. J. Exp. Bot., 35, 1754-1763.

ISTA, 1979. Manuel pour l'appréciation des plantules. Ed. Bekendam J. et Grob R., Zurich, 130 p.

Le Deunff Y., 1971. Action spécifique du potassium sur l'élongation des plantules étiolées de quelques dicotylédones. C.R. Acad. Sci., Paris, Série D, 272, 1644-1647.

Leigh R. A., Wynjones R. G., 1984. A hypothesis relating critical potassium concentrations for growth to the distribution and functions of this ion in the plant cell. New Phytol., 97, 1-13.

Long S. R., Dale R. M. K., Sussex I. M., 1981. Maturation and germination of Phaseolus vulgaris embryonic axes in culture. Planta, 153, $405-415$.

Loomis E. L., Smith O. E., 1980. The effect of artificial aging on the concentration of $\mathrm{Ca}, \mathrm{Mg}, \mathrm{Mn}, \mathrm{K}$ and $\mathrm{Cl}$ in imbibing cabbage seed. J. Am. Soc. Hortic. Sci., 105, 647-650.

Mc Kersie B. D., Stinson R. H., 1980. Effect to dehydration on leakage and membrane structure in Lotus corniculatus L. seeds. Plant Physiol., 66, 316-320.

Misra S., Bewley J. D., 1985. Reprogramming of protein synthesis from a developmental to a germinative mode induced by desiccation of the axes of Phaseolus vulgaris. Plant Physiol., 78, 876-882.

Murray D. R., Kennedy I. R., 1980. Changes in activities of enzymes of nitrogen metabolism in seedcoats and cotyledons during embryo development in pea seeds. Plant Physiol., 66, 782-786.

Obendorf R. L., Wettlaufer S. H., 1984. Precocious germination during in vitro growth of soybean seeds. Plant Physiol, 76, 10231028 .
Pardi D., Morgutti S., Cocucci S., 1980. $\mathrm{K}^{+}$uptake in the early phases of germination of the photoblastic and thermosensitive seed of Phacelia tanacetifolia. Physiol. Plant., 48, 379-384.

Patrick J. W., 1984. Photosynthate unloading from seed coats of Phaseolus vulgaris L. Control by tissue water relations. J. Plant Physiol., 115, 297-310.

Petruzelli L., Lioi L., Garello G., Morgutti S., Cocucei S., 1982. The effect of fusicoccin and monovalent cations on the viability of wheat seeds. J. Exp. Bot., 33, 118-124.

Rachidian Z., Le Deunff Y., 1986. Qualité des semences du pois protéagineux. 1. Développement de la gousse et des graines : incidence sur la germination des semences avec ou sans séchage. Agronomie, 6 (5), 463-468.

Samad I.M.A., Pearce R. S., 1978. Leaching of ions, organic molecules, and enzymes from seeds of peanut (Arachis hypogea L.) imbibing without testas or with intact testas. J. Exp. Bot., 29, 14711478.

Steere W. C., Levengood W. C., Bondie J. M., 1981. An electronic analyser for evaluating seed germination and vigour. Seed Sci. and Technol., 9, 567-576.

Thorne J. H., 1981. Morphology and ultrastructure of maternal seed tissues of soybean in relation to the import of photosynthate. Plant Physiol., 67, 1016-1025.

Thorne J. H., 1982. Characterization of the active sucrose transport system of immature soybean embryos. Plant Physiol., 70, 953958.

Thorne J. H., Rainbird R. M., 1983. An in vivo technique for the study of phloem unloading in seed coats of developing soybean seeds. Plant Physiol., 72, 268-271.

Touboul C., 1984. Contributions à la connaissance des mécanismes de lessivage des semences de lupin blanc (Lupinus albus L.) au cours du trempage et leurs liaisons possibles avec la qualité. Mém. D.E.A. Paris VI, $58 \mathrm{p}$.

Wolswinkel P., Ammerlaan A., 1983. Phloem unloading in developing seeds of Vicia faba $\mathrm{L}$. The effect to several inhibitors on the release of sucrose and amino acids by the seed coat. Planta, 158, 205-215.

Wolswinkel P., Ammerlaan A., 1985. Characteristics of sugar, amino acid and phosphate release from the seed coat of developing seeds of Vicia faba and Pisum sativum. J. Exp. Bot., 36, 359-368. 